\title{
Detecting directional coupling in the human epileptic brain: Limitations and potential pitfalls
}

\author{
Hannes Osterhage, ${ }^{1,2, *}$ Florian Mormann, ${ }^{1,3}$ Tobias Wagner, ${ }^{1,2}$ and Klaus Lehnertz ${ }^{1,2,4, \dagger}$ \\ ${ }^{1}$ Department of Epileptology, Neurophysics Group, University of Bonn, Sigmund-Freud-Strasse 25, 53105 Bonn, Germany \\ ${ }^{2}$ Helmholtz-Institute for Radiation and Nuclear Physics, University of Bonn, Nussallee 14-16, 53115 Bonn, Germany \\ ${ }^{3}$ Division of Biology, California Institute of Technology, 1200 East California Boulevard, Pasadena, California 91125, USA \\ ${ }^{4}$ Interdisciplinary Center for Complex Systems, University of Bonn, Römerstrasse 164, 53117 Bonn, Germany
}

(Received 4 April 2007; revised manuscript received 4 June 2007; published 22 January 2008)

\begin{abstract}
We study directional relationships - in the driver-responder sense-in networks of coupled nonlinear oscillators using a phase modeling approach. Specifically, we focus on the identification of drivers in clusters with varying levels of synchrony, mimicking dynamical interactions between the seizure generating region (epileptic focus) and other brain structures. We demonstrate numerically that such an identification is not always possible in a reliable manner. Using the same analysis techniques as in model systems, we study multichannel electroencephalographic recordings from two patients suffering from focal epilepsy. Our findings demonstrate thatdepending on the degree of intracluster synchrony-certain subsystems can spuriously appear to be driving others, which should be taken into account when analyzing field data with unknown underlying dynamics.
\end{abstract}

DOI: 10.1103/PhysRevE.77.011914 PACS number(s): 87.19.L-, 05.45.Xt, 05.45.Tp, 05.10. -a

\section{INTRODUCTION}

Synchronization was initially described by Huygens as early as in the 17 th century [1]. More recent developments in the theory of dynamical systems has led to numerous investigations of these phenomena [2-10]. It is now well-known that interactions between dynamical systems play an important role in numerous fields of the physical sciences such as electronics [11-13], laser physics [14-16], solid state physics [17], plasma physics [18], communication [19,20], or in control theory $[21,22]$. Furthermore, synchronization has been observed in numerous biological systems. Two well-known examples are the human cardiorespiratory system [23] and the flashing of fireflies [24]. In particular, synchronization plays an important role on a variety of spatial as well as temporal scales in one of the most challenging dynamical systems in nature, namely, the human brain (for an overview, see, e.g., $[25,26]$ and references therein).

The multitude of observed synchronization phenomena has led to the proposition of a number of different frameworks for their description. In the most simple case of complete synchronization the systems' states become identical. This concept can be extended to the case of lag synchronization where the states of the systems coincide if one is shifted in time [27]. Phase synchronization is a relatively new notion that extended the classical notion of synchrony understood as phase and frequency locking to the case of chaotic systems [28]. A completely different approach was used to introduce the framework of generalized synchronization [7,29] for the case when the states of the systems are related through some function that maps the state spaces onto each other.

When investigating synchronization phenomena between dynamical systems, the analysis can be divided into two main aspects. The first question is whether coupling is

\footnotetext{
*h.osterhage@web.de

†klaus.lehnertz@ukb.uni-bonn.de
}

present between the systems and how strong it is, while the second one relates to the causal relationships between the systems, i.e., the direction or asymmetry of the coupling. Both strength and direction cannot be viewed in a completely independent manner (e.g., in the case of complete synchronization where it is not possible to detect any directionality in the coupling, even if it is strictly unidirectional). While a number of time series analysis techniques have been proposed that aim at investigating either the strength [30-33] or the direction [34-42] of interactions, studies that take into account both aspects are notable exceptions $[42,43]$.

A further question of great interest that was addressed in recent studies [44-49] is whether bivariate time series analysis techniques are suited to study global interactions in multivariate data. In the simple case of two systems, the detection of interactions between them is straightforward to interpret. In contrast, when three or more (sub)systems are involved, the situation becomes more complicated. Consider a driving system $D$ and two responding systems $R_{1}$ and $R_{2}$. Let, on the one hand $D$ and $R_{1}$ be synchronized and, on the other hand, $D$ and $R_{2}$ be synchronized as well. In this situation, $R_{1}$ and $R_{2}$ may show synchronized behavior although no coupling is present between them. It is clear that similar difficulties arise when investigating causal relationships. Consider the case when $D$ and $R_{1}$ are so strongly coupled that they show synchronized behavior, while a weaker yet significant coupling is present between $D$ and $R_{2}$, in the sense that the driving is detectable. In that case, not only $D$ but $R_{1}$ may also wrongly be interpreted as driving $R_{2}$. From these considerations arises naturally the question to what extent the detectability of driver-responder relationships in networks of dynamical systems is affected by the strength of the involved interactions. Different studies have already revealed that this detectability is closely related to the level of synchrony between systems $[37,50]$. As a consequence, it has been proposed that results should be interpreted with care if the level of synchrony between systems, which is usually quantified by some measure, exceeds a critical threshold [51]. When studying directionality in time series from systems with unknown underlying dynamics, it is of great im- 
portance to know whether the interplay between the global level of synchrony and the strength of driving only limits detectability, or whether it may also lead to spurious detections of driving. We here address this issue by analyzing time series from model systems and electroencephalographic time series from epilepsy patients.

This paper is organized as follows. In Sec. II, we briefly describe two bivariate approaches from time series analysis, namely, the mean phase coherence [33] and the directionality index [37] as measures for the strength and the direction of interactions, respectively. From the examples we mentioned, it is clear that the problem is of principal nature and independent of the mathematical framework. We thus restrict ourselves to the analysis of phase dynamics. In Sec. III, we introduce the model systems used and present our findings. We show that for certain asymmetrical coupling parameters, spurious driving of oscillators that are coupled to driving subsystems can be detected. We further demonstrate to what extent this behavior is dependent on the strength of the synchronization with the drivers and provide a method to avoid misinterpretations when analyzing systems with unknown dynamics. In Sec. IV, we present findings from analyzing time series of brain electrical activity recorded in patients with focal epilepsy. We finally discuss our results in the concluding section.

\section{METHODS}

\section{A. Mean phase coherence}

The mean phase coherence [33] is a measure for the strength of the synchronization between two dynamical systems. It is derived from the phase locking condition that is fulfilled when the systems under investigation are synchronized,

$$
\left|m \phi_{1}(t)-n \phi_{2}(t)\right| \leq \mathrm{const}, \quad m, n \in \mathbb{N},
$$

where $\phi_{1}(t)$ and $\phi_{2}(t)$ are the phase variables of systems $S_{1}$ and $S_{2}$, respectively. We here restrict ourselves to the case of $m=n=1$ and derive the phase variables from an analytical signal approach $[52,53]$. For time series of length $N$, the degree of synchronization can be determined from the circular distribution of the measured phase differences $[\delta \phi(j)$ $\left.=\phi_{1}(j \Delta t)-\phi_{2}(j \Delta t)\right]_{j=1, \ldots, N}$ using the mean phase coherence $R$,

$$
R=\left|\frac{1}{N} \sum_{j=1}^{N} \exp i \delta \phi(j)\right|,
$$

with $\Delta t$ denoting the sampling interval. $R$ is confined to the interval $[0,1]$ where $R=1$ indicates fully synchronized systems.

\section{B. Directionality index}

The directionality index [37] as a measure for the direction of interactions between two dynamical systems is based on modeling the evolution of the unwrapped phase time series $\left[\phi_{1,2}(j \Delta t)=\phi_{1,2}^{(j)}\right]_{j=1, \ldots, N}$ of systems $S_{1}$ and $S_{2}$. The phase increments $\left[\Omega_{1,2}(j)=\phi_{1,2}^{(j+\tau)}-\phi_{1,2}^{(j)}\right]_{j=1, \ldots, N-\tau}$ over some fixed time $\tau$ are considered to be generated by unknown twodimensional maps,

$$
\Omega_{1,2}(j)=\mathbb{F}_{1,2}\left[\phi_{1,2}^{(j)}, \phi_{2,1}^{(j)}\right]+\eta_{1,2},
$$

with the random terms $\eta_{1,2}$ representing noisy perturbations. This rather intuitive approach does not require explicit knowledge of the dynamics governing the systems. This is of great importance when studying field data, since there are often no realistic models available, which would allow a more reliable estimation of strength and direction of coupling (see, e.g., Refs. [43,54]). We followed Ref. [50] and used $\tau=\min \left(T_{1}, T_{2}\right), T_{1}$ and $T_{2}$ being the periods of oscillation of the systems $S_{1}$ and $S_{2}$, respectively. These maps can be approximated using finite Fourier series

$$
F_{1,2}\left(\phi_{1,2}, \phi_{2,1}\right)=\sum_{k, l} A_{1,2}^{(k l)} \exp \left(i k \phi_{1,2}+i l \phi_{2,1}\right) .
$$

The coefficients $A_{1,2}^{(k l)}$ are obtained by solving the linear leastsquares problems $\mathbb{F}_{1,2}^{2} \approx F_{1,2}$, thus obtaining estimates for the deterministic parts of the maps. We followed Ref. [37] and used the following terms: $|l| \leq 3$ for $k=0,|k| \leq 3$ for $l=0$, and $k=l=1$.

The influence of the systems on each other are now quantified by terms $c_{1,2}$ which are defined as

$$
c_{1,2}^{2}=\int_{0}^{2 \pi} \int_{0}^{2 \pi}\left(\frac{\partial F_{1,2}}{\partial \phi_{2,1}}\right)^{2} d \phi_{1} d \phi_{2} .
$$

The directionality of the coupling between the systems is quantified as

$$
d^{(1,2)}=\frac{c_{2}-c_{1}}{c_{1}+c_{2}} .
$$

$d^{(1,2)}$ is expected to vary between 1 for unidirectional coupling with $S_{1}$ as the driver and -1 for the opposite case. $d^{(1,2)}=0$ is expected for symmetric bidirectional coupling.

Since we study networks of coupled dynamical systems we substitute $R^{(i, j)}$ for $R$ and $d^{(i, j)}$ for $d^{(1,2)}$ throughout the remainder of this paper.

\section{APPLICATION TO MODEL SYSTEMS}

Our aim is to evaluate the traceability of directional coupling in systems with unknown underlying dynamics. A wellknown example for such a system is the human brain, in which high levels of synchrony can be observed between structurally similar brain regions. Since directionality cannot be detected in fully synchronized systems [37,50], an important question is to what degree directional coupling can be detected in the human brain given that neighboring structures already exhibit a high level of synchrony. To this end we here study systems with nearest-neighbor couplings that allow one to simulate clusters of interacting oscillators with different levels of intercluster and intracluster synchrony.

We here used a network of 20 diffusively coupled Rössler oscillators [55] for our simulations, each of which obeys the following equations of motion:

$$
\dot{x}^{(k)}=-\omega^{(k)} y^{(k)}-z^{(k)}+\xi_{C}^{(k)}+\xi_{D}^{(k)},
$$




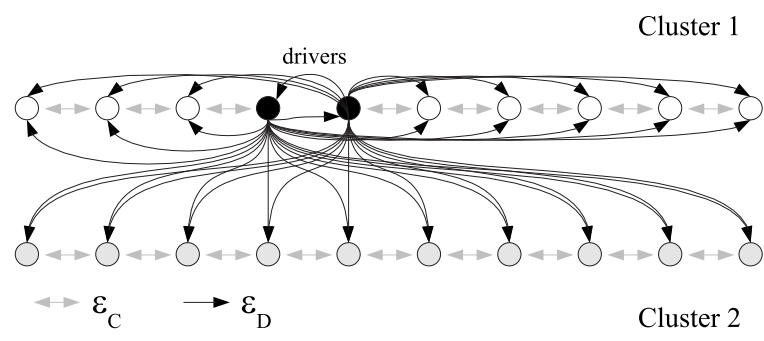

FIG. 1. Coupling scheme used in the presented work. The upper and lower rows represent oscillators from cluster $C_{1}$ (white, responders; black, drivers) and from cluster $C_{2}$ (gray), respectively. Black and gray arrows symbolize unidirectional $\left(\epsilon_{D}\right)$ and bidirectional $\left(\epsilon_{C}\right)$ couplings, respectively.

$$
\begin{aligned}
& \dot{y}^{(k)}=\omega^{(k)} x^{(k)}+0.165 y^{(k)}, \\
& \dot{z}^{(k)}=0.2+z^{(k)}\left(x^{(k)}-10\right),
\end{aligned}
$$

with $k=1, \ldots, 20$ denoting the different oscillators $X_{k}$ with frequencies $\omega^{(k)}$, and bidirectional and unidirectional coupling terms $\xi_{C}^{(k)}$ and $\xi_{D}^{(k)}$, respectively. We divided the network into two clusters $C_{1}=\left\{X_{1}, \ldots, X_{10}\right\}$ and $C_{2}=\left\{X_{11}, \ldots, X_{20}\right\}$ of 10 oscillators each. Within the clusters, neighboring oscillators are coupled bidirectionally with strength $\epsilon_{C}$. In addition we introduced two driving systems $\left\{X_{4}, X_{5}\right\}$ which we coupled unidirectionally to all other oscillators with strength $\epsilon_{D}$. The setting is illustrated in Fig. 1. The coupling terms $\xi_{C}^{(k)}$ and $\xi_{D}^{(k)}$ are given by

$$
\begin{gathered}
\xi_{C}^{(k)}= \begin{cases}\epsilon_{C}\left(x^{(k+1)}-x^{(k)}\right), & k=\{1,5,11\}, \\
\epsilon_{C}\left(x^{(k-1)}-x^{(k)}\right), & k=\{4,10,20\}, \\
\epsilon_{C}\left(x^{(k+1)}+x^{(k-1)}-2 x^{(k)}\right), & \text { otherwise, }\end{cases} \\
\xi_{D}^{(k)}=\epsilon_{D}\left(x^{(4)}+x^{(5)}-2 x^{(k)}\right) .
\end{gathered}
$$

The global behavior of the network is thus controlled by the two parameters $\epsilon_{D}$ and $\epsilon_{C}$. This allows us to investigate the detectability of directional coupling $\epsilon_{D}$ for different levels of local bidirectional coupling $\epsilon_{C}$, which in turn can be used to control the global degree of synchrony within the clusters.

The equations of motion were solved using a fourth-order Runge-Kutta integration method with a step size of 0.05 and a sampling interval $\Delta t=0.1$. Initial conditions were randomly chosen in the state space near the Rössler attractor for each oscillator and realization separately. In order to eliminate transients, the first $10^{4}$ iterations were discarded.

We focus on the applicability of time series analysis techniques to field data and thus restrict ourselves to time series consisting of 4096 data points. In order to eliminate frequency effects when the systems are uncoupled, we randomly chose the frequencies $\omega^{(k)}$ from a Gaussian distribution with mean of $\bar{\omega}=0.9$ and standard deviation $\sigma=0.1$. In another study [56] we have shown that the directionality index is affected by parameter mismatches of systems under investigation. To avoid biased values of $d^{(i, j)}$ that are trivially caused by different eigenfrequencies of the subsystems, we generated $N_{\alpha}=100$ different solutions of the equations of motion for each pair $\left\{\epsilon_{D}, \epsilon_{C}\right\}$. For each realization $\alpha$ $=1, \ldots, N_{\alpha}$, a new random set of frequencies $\omega^{(k)}$ was generated in order to obtain mean values $\bar{\omega}=0.9$ not only across the network but also for each oscillator separately.

For each realization $\alpha$, we calculated both the mean phase coherence and the directionality index for all possible pairs of oscillators, thus obtaining matrices $\mathbf{R}^{(\alpha)}\left(\epsilon_{C}, \epsilon_{D}\right), \mathbf{D}^{(\alpha)}$ $\times\left(\epsilon_{C}, \epsilon_{D}\right), \alpha=1, \ldots, N_{\alpha}$ with entries $R_{i j}^{(\alpha)}=R^{(i, j)}\left(\epsilon_{C}, \epsilon_{D}, \alpha\right)$, $D_{i j}^{(\alpha)}=d^{(j, i)}\left(\epsilon_{C}, \epsilon_{D}, \alpha\right)$ corresponding to combinations $\left\{X_{i}, X_{j}\right\}$, $i, j=1, \ldots, 20$. Averaging over all realizations yielded matrices

$$
\begin{aligned}
& \mathbf{R}\left(\epsilon_{C}, \epsilon_{D}\right)=\frac{1}{N_{\alpha}} \sum_{\alpha=1}^{N_{\alpha}} \mathbf{R}^{(\alpha)}\left(\epsilon_{C}, \epsilon_{D}\right), \\
& \mathbf{D}\left(\epsilon_{C}, \epsilon_{D}\right)=\frac{1}{N_{\alpha}} \sum_{\alpha=1}^{N_{\alpha}} \mathbf{D}^{(\alpha)}\left(\epsilon_{C}, \epsilon_{D}\right) .
\end{aligned}
$$

By definition $\mathbf{R}$ is symmetric while $\mathbf{D}$ is antisymmetric. Note that we use the convention that $\mathbf{D}_{i j}>0$ is expected if $X_{j}$ is driving $X_{i}$.

To describe the synchronization phenomena on the scale of the two clusters we define internal and external synchronization strengths $R_{I}$ and $R_{E}$ (corresponding to intracluster and intercluster synchronization, respectively),

$$
\begin{gathered}
R_{I}\left(\epsilon_{C}, \epsilon_{D}\right)=\frac{1}{\left.M_{I\left\{i, j \mid X_{i}\right.} \in C_{q}, X_{j} \in C_{q}, i \neq j\right\}_{q=1,2}} R_{i j}\left(\epsilon_{C}, \epsilon_{D}\right), \\
R_{E}\left(\epsilon_{C}, \epsilon_{D}\right)=\frac{1}{M_{E}} \sum_{\left\{i, j \mid X_{i} \in C_{1}, X_{j} \in C_{2}\right\}} R_{i j}\left(\epsilon_{C}, \epsilon_{D}\right) .
\end{gathered}
$$

$M_{I}$ and $M_{E}$ are equal to the corresponding number of summands. $R_{I}\left(\epsilon_{C}, \epsilon_{D}\right)$ is the average mean phase coherence for combinations of oscillators that are elements of the same cluster and is thus expected to increase when $\epsilon_{C}$ is increased. In contrast, $R_{E}\left(\epsilon_{C}, \epsilon_{D}\right)$ is the average mean phase coherence for combinations of oscillators that belong to different clusters and is expected to increase with increasing $\epsilon_{D}$. Furthermore, we define the mean local directionality index of the drivers, $D_{D}\left(\epsilon_{C}, \epsilon_{D}\right)$, and the global intercluster mean directionality index, $D_{G}\left(\epsilon_{C}, \epsilon_{D}\right)$,

$$
\begin{gathered}
D_{D}\left(\epsilon_{C}, \epsilon_{D}\right)=\frac{1}{M_{D}} \sum_{i \neq 4, i \neq 5} D_{i 4}\left(\epsilon_{C}, \epsilon_{D}\right)+D_{i 5}\left(\epsilon_{C}, \epsilon_{D}\right), \\
D_{G}\left(\epsilon_{C}, \epsilon_{D}\right)=\frac{1}{M_{G}} \sum_{\left\{i, j \mid X_{j} \in C_{1}, X_{i} \in C_{2}, j \neq 4, j \neq 5\right\}} D_{i j}\left(\epsilon_{C}, \epsilon_{D}\right) .
\end{gathered}
$$

In analogy to Eq. (10), $M_{D}$ and $M_{G}$ equal the corresponding number of summands. $D_{D}\left(\epsilon_{C}, \epsilon_{D}\right)$ is the average directionality index for all possible combinations of any of the two drivers with responding oscillators and should thus increase with increasing $\epsilon_{D} . D_{G}\left(\epsilon_{C}, \epsilon_{D}\right)$ is the averaged directionality index for all combinations of responders $\left\{X_{i}, X_{j}\right\}$ with $X_{i} \in C_{1}$ and $X_{j} \in C_{2} . D_{G}\left(\epsilon_{C}, \epsilon_{D}\right)>0$ indicates a predominant driving 


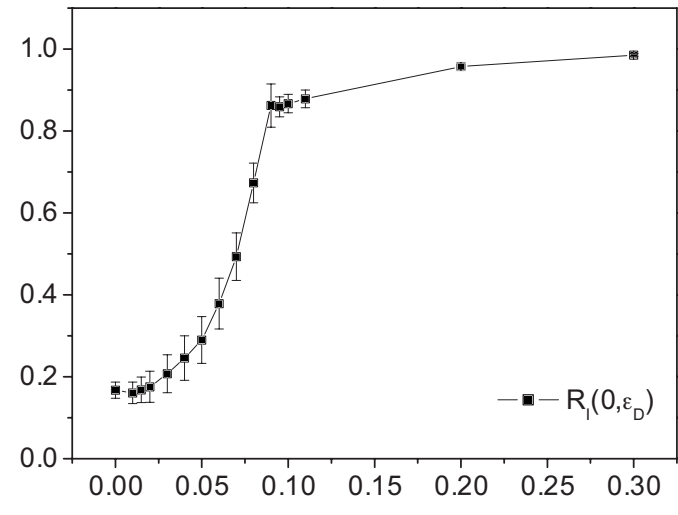

(a)

$\varepsilon_{\mathrm{D}}$

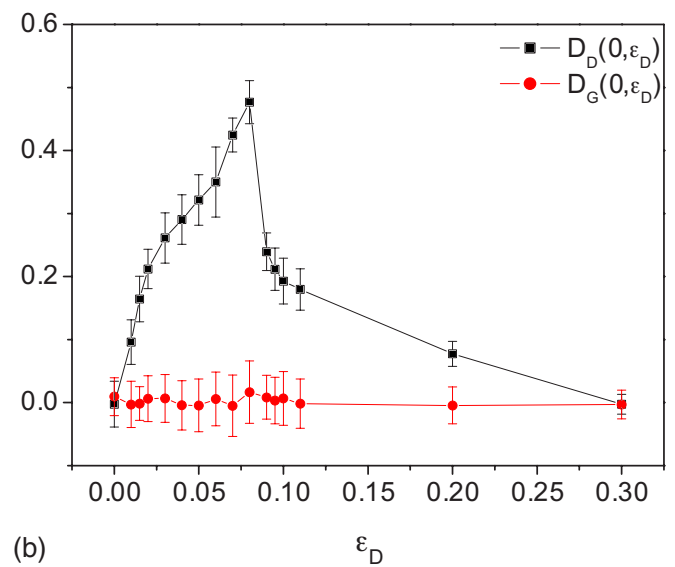

FIG. 2. (Color online) Dependence of $R_{I}$ (a) and of $D_{D}$ and $D_{G}$ (b) on the driver coupling strength $\epsilon_{D}$ for $\epsilon_{C}=0$. Error bars denote standard deviation over different pairs of oscillators.

of oscillators in $C_{2}$ by oscillators in $C_{1}$ while $D_{G}\left(\epsilon_{C}, \epsilon_{D}\right)$ $<0$ would indicate the opposite situation.

The influence of $\epsilon_{D}$ on the dynamical behavior of the network is shown in Fig. 2 for $\epsilon_{C}=0$, i.e., when no intercluster coupling is present and all 20 oscillators are driven by oscillators $X_{4}$ and $X_{5} \cdot R_{I}\left(0, \epsilon_{D}\right) \approx R_{E}\left(0, \epsilon_{D}\right)$ follows from the fact that no cluster structures are present in this case. Since $R_{I}\left(0, \epsilon_{D}\right)$ and $R_{E}\left(0, \epsilon_{D}\right)$ exhibit almost identical dependence on $\epsilon_{D}$, we only show the behavior of $R_{I}\left(0, \epsilon_{D}\right)$ in Fig. 2(a). Low variances of $R_{I}\left(0, \epsilon_{D}\right)$ and $R_{E}\left(0, \epsilon_{D}\right)$ over the different combinations of oscillators clearly indicates the same degree of synchronization between all oscillators for different $\epsilon_{D}$.

Figure 2(b) shows the different behaviors of $D_{D}\left(0, \epsilon_{D}\right)$ and $D_{G}\left(0, \epsilon_{D}\right) . D_{G}\left(0, \epsilon_{D}\right) \approx 0$ independently of $\epsilon_{D}$. The low variances over combinations of oscillators indicates that $D_{i j}\left(0, \epsilon_{D}\right) \approx$ const for all $\{i, j\}$. The directionality index therefore correctly reflects the fact that there is symmetric coupling present between the driven oscillators. From the course of $D_{D}\left(0, \epsilon_{D}\right)$ it is obvious that the directionality index $d^{(i, j)}$ depends not only on the asymmetry of the coupling but also on its absolute value. Starting at $D_{D}\left(0, \epsilon_{D}\right)=0$ for completely independent systems, $D_{D}\left(0, \epsilon_{D}\right)$ increases monotonically until a maximum is reached at $\epsilon_{D}=0.08$, which reflects the growing influence of the drivers on the dynamics of the re- sponders. However, for $\epsilon_{D}>0.08$ a weakly synchronized state is reached $\left[R_{I}\left(0, \epsilon_{D}\right) \approx\right.$ const for $\left.\epsilon_{D}>0.08\right]$. At $\epsilon_{D}$ $\approx 0.3$ the oscillators become fully synchronized $\left[R_{I}\left(0, \epsilon_{D}\right)\right.$ $\approx 1]$. Consequently, the coupling direction cannot be detected here. Note that the transition from the maximal value of $D_{D}\left(0, \epsilon_{D}\right)$ to $D_{D}\left(0, \epsilon_{D}\right) \approx 0$ is not abrupt but rather occurs over a range of values of $\epsilon_{D}$, reflecting the smooth adaption of the responders to the drivers' dynamics.

The dependencies of $R_{I}\left(\epsilon_{C}, \epsilon_{D}\right)$ and $R_{E}\left(\epsilon_{C}, \epsilon_{D}\right)$ on the cluster coupling strength $\epsilon_{C}$ are shown in Fig. 3 for weak (a), intermediate (b), and strong (c) driver coupling strengths $\epsilon_{D}$. For weak driving [Fig. 3(a)] no synchronization between $C_{1}$ and $C_{2}$ is observed, and $R_{E}\left(\epsilon_{C}, \epsilon_{D}\right)$ increases only slightly when $\epsilon_{C}$ is increased. Accordingly, in the matrix $\mathbf{R}(0.07,0.01)$ entries that correspond to combinations of oscillators from different clusters assume low and almost constant values. In contrast, the intracluster synchronization $R_{I}\left(\epsilon_{C}, \epsilon_{D}\right)$ increases substantially, with a high variance across the different combinations of oscillators. Since nearestneighbor coupling is present, the mean phase coherence between neighboring oscillators is high while remote oscillators within one cluster remain independent and correspondingly the mean phase coherence is low.

For intermediate driving $\epsilon_{D}$ [Fig. 3(b)], the variance of $R_{I}\left(\epsilon_{C}, \epsilon_{D}\right)$ is clearly decreased when compared to the variances for small $\epsilon_{D}$. This can be attributed to the fact that synchronization between remote oscillators within one cluster is increased through the increase of $\epsilon_{D}$. Furthermore, the increase of $R_{E}\left(\epsilon_{C}, \epsilon_{D}\right)$ is more pronounced since the clusters are now more strongly coupled through the drivers. This is reflected in the corresponding matrix $\mathbf{R}(0.065,0.03)$ : in contrast to Fig. 3(a), increased values of $R_{i j}\left(\epsilon_{C}, \epsilon_{D}\right)$ are not only observed within the clusters but also in combinations of oscillators from different clusters. Since entries $R_{i j}\left(\epsilon_{C}, \epsilon_{D}\right)$ that correspond to intercluster combinations of oscillators only depend on the global driving $\epsilon_{D}$ their variances are low when compared to the variance of entries corresponding to intracluster combinations.

A further increase of $\epsilon_{D}$ to 0.06 results in a globally synchronized network for all but very low $\epsilon_{C}$ [Fig. 3(c)]. $R_{I}\left(\epsilon_{C}, \epsilon_{D}\right)$ and $R_{E}\left(\epsilon_{C}, \epsilon_{D}\right)$ are almost identical. As is shown in the corresponding matrix $\mathbf{R}(0.1,0.06)$, it becomes almost impossible to discriminate the clusters from each other.

We now turn to the dependencies of $D_{D}\left(\epsilon_{C}, \epsilon_{D}\right)$ and $D_{I}\left(\epsilon_{C}, \epsilon_{D}\right)$ for the same pairs of control parameters $\left\{\epsilon_{C}, \epsilon_{D}\right\}$ (cf. Fig. 4). For weak driving $\left[\epsilon_{D}=0.01\right.$, cf. Fig. 4(a) $]$ $D_{D}\left(\epsilon_{C}, \epsilon_{D}\right)$ initially increases before it slightly decays to almost constant values $\left(\epsilon_{C}>0.08\right)$. The initial increase can be explained by an increasingly coherent driving from both $X_{4}$ and $X_{5}$. At first sight this appears contradictory since these oscillators are only coupled through $\epsilon_{D}$, which is constant. However, an increase of the cluster coupling strength $\epsilon_{C}$ causes the mean phase coherence between the drivers to increase. The reason for this is that the drivers are indirectly coupled to each other through the bidirectional coupling to their nondriving nearest neighbors (Fig. 1). For $\epsilon_{C}>0.08$ a considerably increased variance of $D_{D}\left(\epsilon_{C}, \epsilon_{D}\right)$ is observed. Simultaneously an increase of $D_{G}\left(\epsilon_{C}, \epsilon_{D}\right)$ is seen with comparatively low variance. For an intermediate driving level 
a)

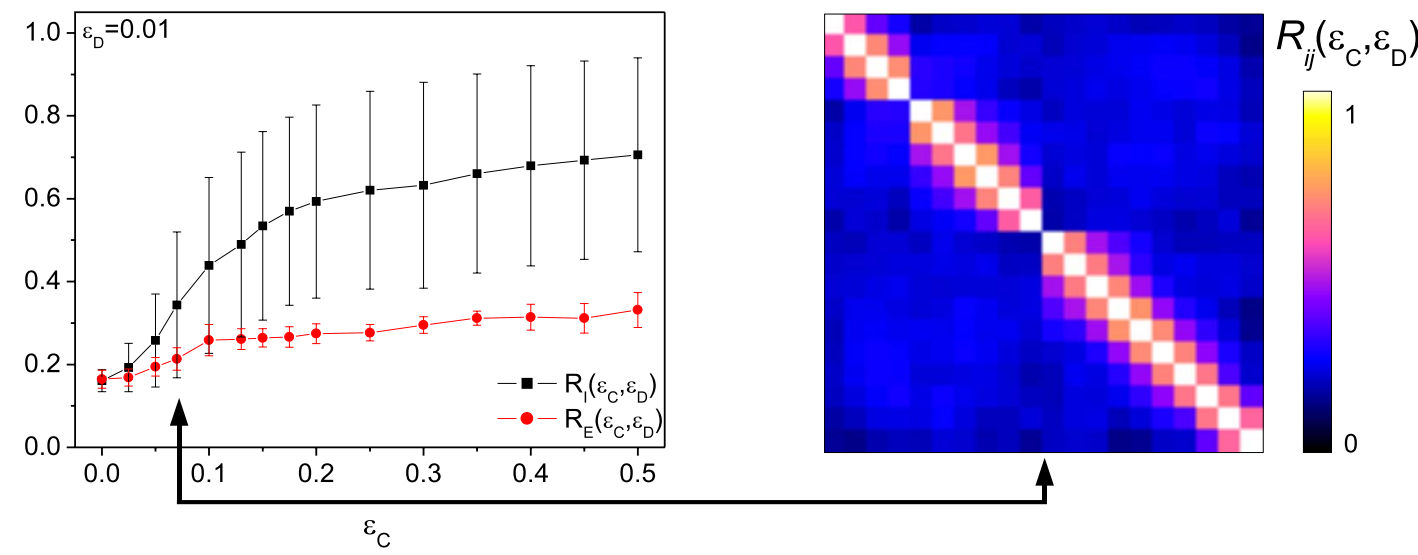

b)

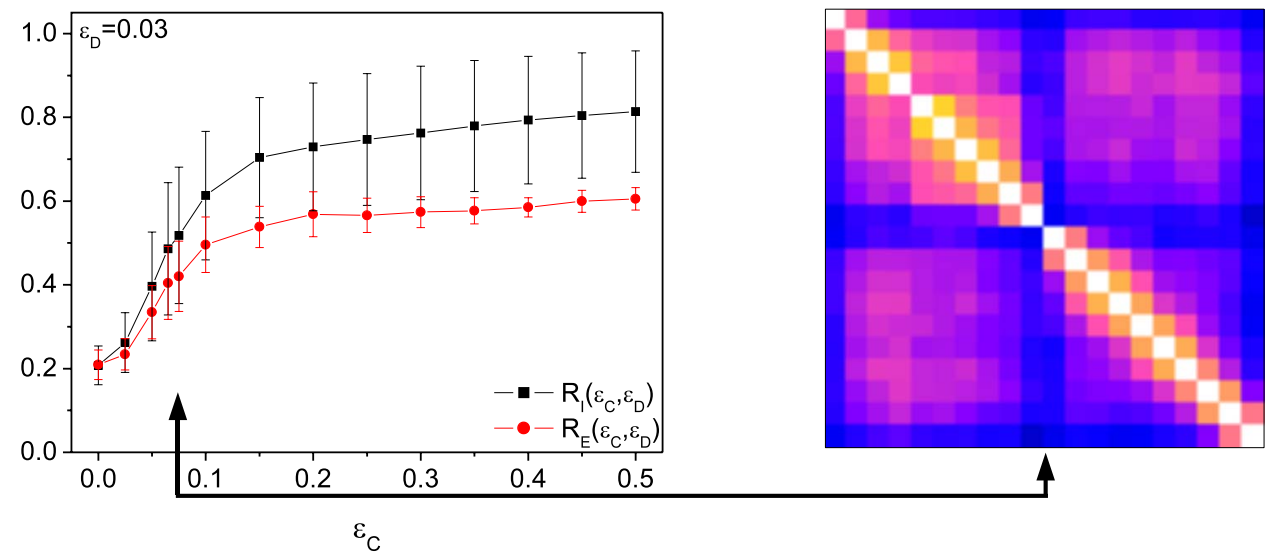

c)

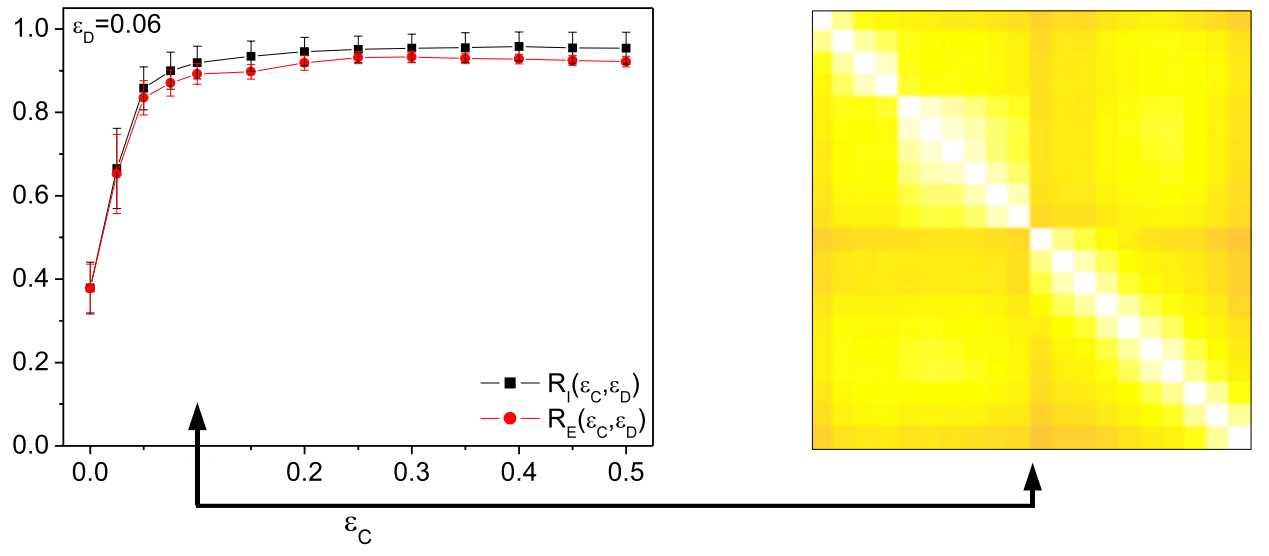

FIG. 3. (Color online) Left-hand side: Dependence of $R_{I}\left(\epsilon_{C}, \epsilon_{D}\right)$ and $R_{E}\left(\epsilon_{C}, \epsilon_{D}\right)$ on $\epsilon_{C}$ for weak $\left[\epsilon_{D}=0.01\right.$ (a)], intermediate $\left[\epsilon_{D}=0.03\right.$ (b)], and strong $\left[\epsilon_{D}=0.06\right.$ (c) $]$ driving. Error bars denote standard deviation over different pairs of oscillators. Right-hand side: For each case, an exemplary matrix $\mathbf{R}\left(\epsilon_{C}, \epsilon_{D}\right)$ is shown for a cluster coupling strength of $\epsilon_{C}=0.07$ (a), $\epsilon_{C}=0.065$ (b), and $\epsilon_{C}=0.1$ (c), respectively.

$\epsilon_{D}=0.03[$ Fig. 4(b)] one observes similar dependencies on $\epsilon_{C}$, except that the initial increase of $D_{D}\left(\epsilon_{C}, \epsilon_{D}\right)$ becomes less pronounced. Finally, for a strong driving level $\epsilon_{D}=0.06$ [Fig. 4(c)] the initial increase completely disappears and a rapid decay of $D_{D}\left(\epsilon_{C}, \epsilon_{D}\right)$ to values around zero is observed. In contrast, $D_{G}\left(\epsilon_{C}, \epsilon_{D}\right)$ remains stable at values around zero. This follows from the previously described phenomenon that a detection of coupling direction is not possible in synchronized systems [cf. Fig. 4(c)]. Even more remarkable is the increase of $D_{G}\left(\epsilon_{C}, \epsilon_{D}\right)$ for weak and intermediate coupling. If the dynamics were unknown this would suggest that $C_{2}$ is globally driven by $C_{1}$, although in this case only two oscil- lators within $C_{1}$ are driving the other oscillators contained in both $C_{2}$ and $C_{1}$. In order to investigate this in a more detailed manner we show matrices $\mathbf{D}\left(\epsilon_{C}, \epsilon_{D}\right)$ for $\epsilon_{D}=0.03$ and increasing $\epsilon_{C}$ in Fig. 4(d). For low values of the cluster coupling strength $\left(\epsilon_{C}=\{0,0.015\}\right) X_{4}$ and $X_{5}$ are correctly identified as driving all other systems. For $\epsilon_{C}=0.04$ a slight overall increase is observed in the apparent driving of $C_{2}$ by $C_{1}$ (lower left corner of the corresponding matrix) while $X_{4}$ and $X_{5}$ can still clearly be identified as the main driving systems. However, when $\epsilon_{C}$ is increased further, a broadening of the apparent driving region is observed, i.e., oscillators coupled to the drivers through $\epsilon_{C}$ are spuriously identified as 
a)

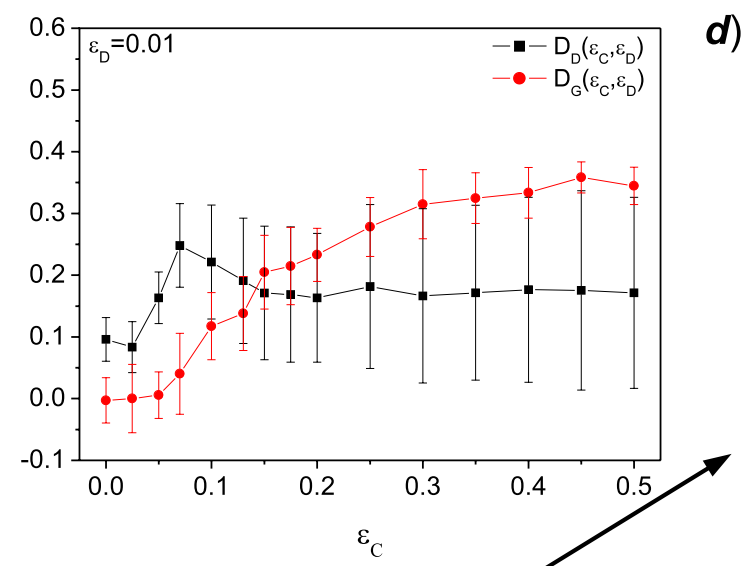

b)

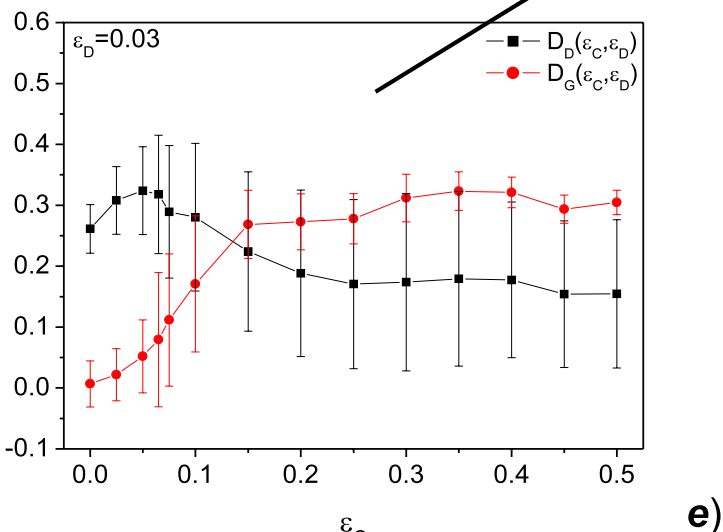

c)

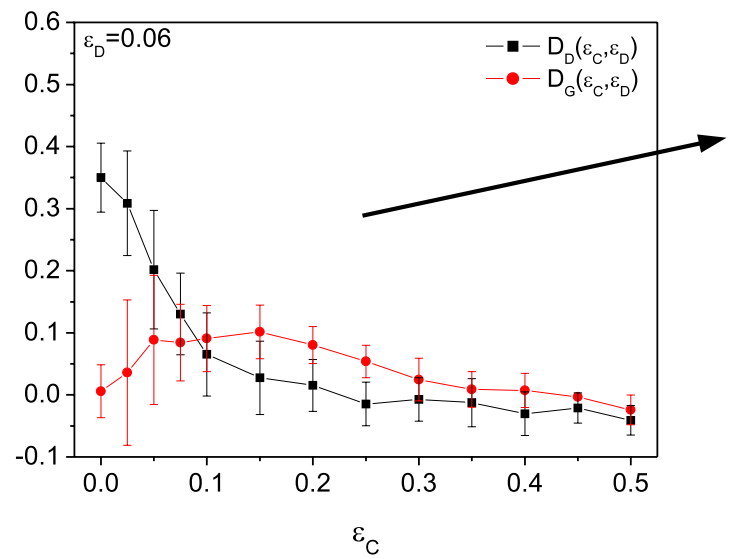

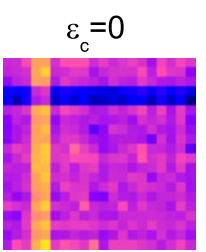
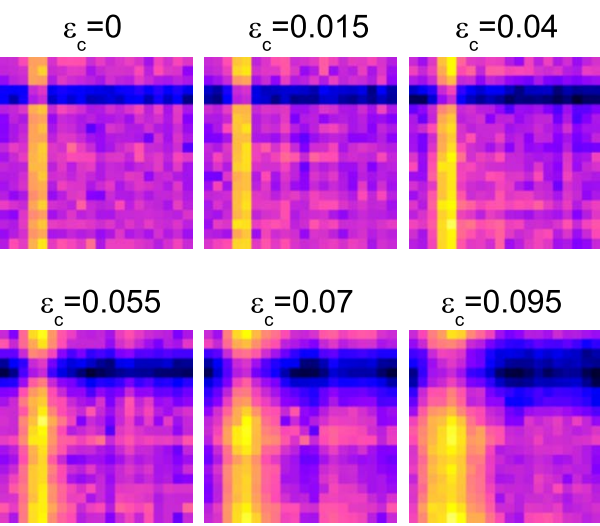

$\varepsilon_{\mathrm{c}}=0.095$

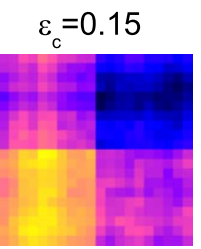

$\varepsilon_{\mathrm{c}}=0.3$

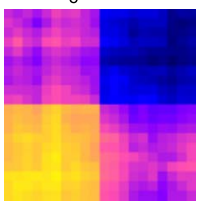

$\varepsilon_{\mathrm{c}}=0.5$

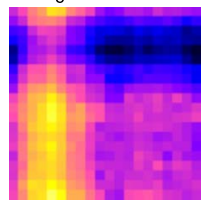

$-0.5$
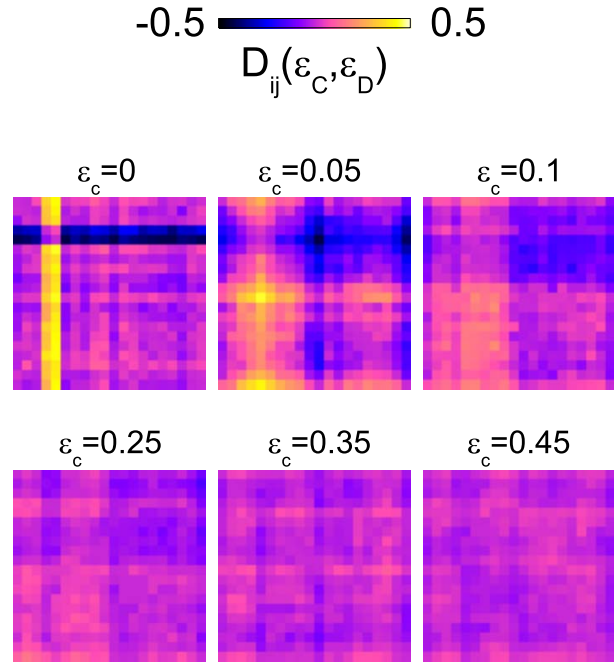

$\varepsilon=0.45$
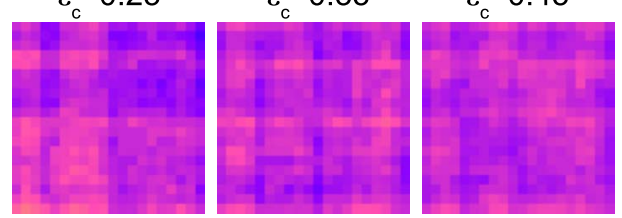

FIG. 4. (Color online) Left-hand side: Dependence of $D_{D}\left(\epsilon_{C}, \epsilon_{D}\right)$ and $D_{G}\left(\epsilon_{C}, \epsilon_{D}\right)$ on $\epsilon_{C}$ for weak $\left[\epsilon_{D}=0.01\right.$ (a)], intermediate $\left[\epsilon_{D}\right.$ $=0.03$ (b) $]$, and strong $\left[\epsilon_{D}=0.06\right.$ (c) $]$ driving. Error bars denote standard deviation over different pairs of oscillators. Right-hand side: For the intermediate (d) and strong (e) drivings, representative examples of corresponding matrices $\mathbf{D}\left(\epsilon_{C}, \epsilon_{D}\right)$ are shown.

additional drivers. As an example, for $\epsilon_{C}=0.095$ a structure consisting of at least four oscillators $\left(X_{3}, X_{4}, X_{5}, X_{6}\right)$ appears to be driving the remaining systems in both clusters. For $\epsilon_{C} \geq 0.015$ a state is reached when the whole cluster $C_{1}$ appears to drive $C_{2}$ (lower right-hand matrix).

Spurious driving can also be observed for higher driving coupling strength $\epsilon_{D}$. As shown in Fig. 4(e), a broadening of the driving region can be observed here as well. However, the detected driving is far less pronounced since the oscillators are synchronized at high values of $\epsilon_{D}$ and $\epsilon_{C}$. The broadening is thus paired with a lowering of the corresponding values of $D_{i j}$. Consequently, only a weak driving can be de- tected for $\epsilon_{C}=0.1$. For $\epsilon_{C}>0.35$ no directional coupling can be detected.

Our findings from model systems thus suggest that it is important to check the global level of synchronization (using the mean phase coherence or a comparable measure for the strength of interaction) to avoid misinterpretations when analyzing systems with unknown dynamics. Results indicating that certain parts of a network are driving other parts should be interpreted with care if intermediate values of the mean phase coherences are observed. If no driving is measured, an inspection of the mean phase coherences between the different systems could help to clarify whether indeed no driving 
is present or whether driving may possibly be present, but remained undetected because the systems are strongly synchronized. Note, that more complex cases may occur where different synchronized states coexist [57]. For such cases, the proposed straightforward analysis of the global level of synchrony may not be appropriate.

\section{APPLICATION TO FIELD DATA}

Synchronization phenomena play an important role in the human brain. The applicability of methods for the detection of the strength of interactions between different brain regions using electroencephalographic (EEG) time series has been demonstrated in a number of cases, yielding meaningful results for such different problems as the detection of changes in the dynamics of the brain before epileptic seizures [33,58-61] or the identification of the seizure generating area of the brain (epileptic focus) [56]. Directionality measures have mostly been applied to investigate the propagation of epileptic seizures from the focal area to other brain regions [62-66], but also for the identification of the epileptic focus [67].

An important and yet unanswered question in epileptology is whether pathological interactions between the epileptic focus and remote areas can be identified during seizurefree periods from patients with focal epilepsy. The detection of such interactions in the driver-responder sense would help to better understand the epileptogenic process. In addition, identification of the epileptic focus as a driving region could improve the exact delineation and selection of the brain area to be resected in epilepsy surgery. Thus, a question of particular interest is whether pathological interactions are locally restricted to the immediate surroundings of the focus or whether they also involve remote brain regions. Our findings from model systems reported above indicate that a localization of the epileptic focus by means of directional interactions could be limited to cases where the degree of synchronization within the network is sufficiently low to avoid the observed broadening of the apparent driving region.

In the following we present findings obtained from investigating synchronization phenomena in intracranial EEG recordings from two patients with mesial temporal lobe epilepsy. In both patients, magnetic resonance imaging revealed a sclerosis of the left hippocampus. Invasive recordings of seizure onsets confirmed this area to be the seizure onset zone. After neurosurgical resection of the hippocampal formation, both patients were seizure free, so in both cases we can assume that the focus was located within the resected region. The EEG was recorded prior to surgery via intrahippocampal depth electrodes (cf. Fig. 5), each equipped with 10 cylindrical contacts (length, $2.5 \mathrm{~mm}$; intercontact distance, $4 \mathrm{~mm}$ ), using an average common reference. These electrodes were implanted stereotactically in the medial temporal lobes. EEG recordings were performed at a sampling frequency of $173.61 \mathrm{~Hz}$ using a $12 \mathrm{bit}$ analog to digital (A/D) converter, and the data was bandpass filtered from 0.85 to 85 $\mathrm{Hz}$ (12 dB/oct). All patients had signed informed consent that their clinical data might be used an published for research purposes, and the study protocol had previously been

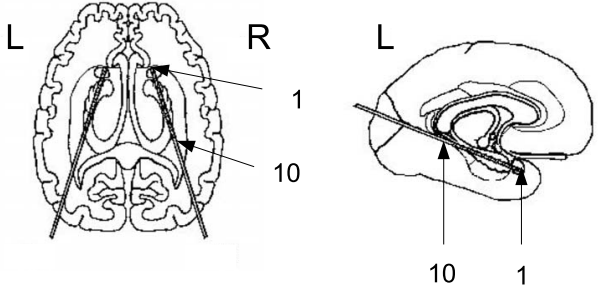

FIG. 5. Schematic view of bilateral intrahippocampal depth electrodes, each equipped with 10 cylindrical contacts of a nickelchromium alloy. Left-hand side, axial view; right-hand side, sagittal view.

approved the local ethics committee. An exemplary recording from patient 1 is shown in Fig. 6. We analyzed recordings from the seizure-free (interictal) period (patient 1, three recordings of different duration, range 40-60 min; patient 2, two recordings of 30 and of $119 \mathrm{~min}$ ). The data were chosen such that each recording started at least 1 hour after a seizure and ended at least $4 \mathrm{~h}$ before a seizure.

We performed the following steps of analysis. As a compromise between the statistical accuracy for the calculation of the measures and the approximate stationarity of the time series, we divided the data into nonoverlapping segments of $23.6 \mathrm{~s}$ duration (corresponding to 4096 data points). This allowed us to calculate both the mean phase coherence and the directionality index for each combination of pairs of electrodes (210 nonredundant pairs per patient) in a moving window fashion with subsequent temporal averaging of the obtained measure values over these windows. We thus obtained a mean phase coherence matrix $\mathbf{R}_{i}$ and a directionality matrix $\mathbf{D}_{i}(i=1,2)$ for each patient. Figure 7 shows the two matrices for patient 1 . The directionality matrix indicates that the structure recorded at the fourth and fifth electrode in the left hemisphere is driving both the left and right mesial temporal regions of the brain. Clinical evaluation of the EEG at seizure onset (not shown here) confirmed the first signs of seizure activity to be restricted to these two contacts. The driving region thus apparently corresponds to the epileptic focus. The mean phase coherence matrix for this patient indicates a low global level of synchronization. Strong coupling appears to be present only in EEG data recorded at neighboring contacts.

The mean phase coherence matrix for patient 2 (Fig. 8) indicates high synchronization in both hemispheres so that the two hemispheres can clearly be identified as separate clusters. Like for patient 1 , the directionality matrix indicates a driving region located within the focal hemisphere. However, it is not possible to clearly circumscribe the driving region since it extends over a structure that was recorded over a range of contacts in the left hemisphere (L5 to L10). For this patient, clinical evaluation of the EEG did not reveal a clearly circumscribed focal area. Here, the first signs of seizure activity were spread over the contacts L5 to L9, in compliance with their high synchronization. According to our findings from model systems (see Sec. III), it is not possible to decide whether there is indeed a broad driving region within the focal hemisphere, or whether there is local driving which appears broadened due to high coupling between the recording sites. 


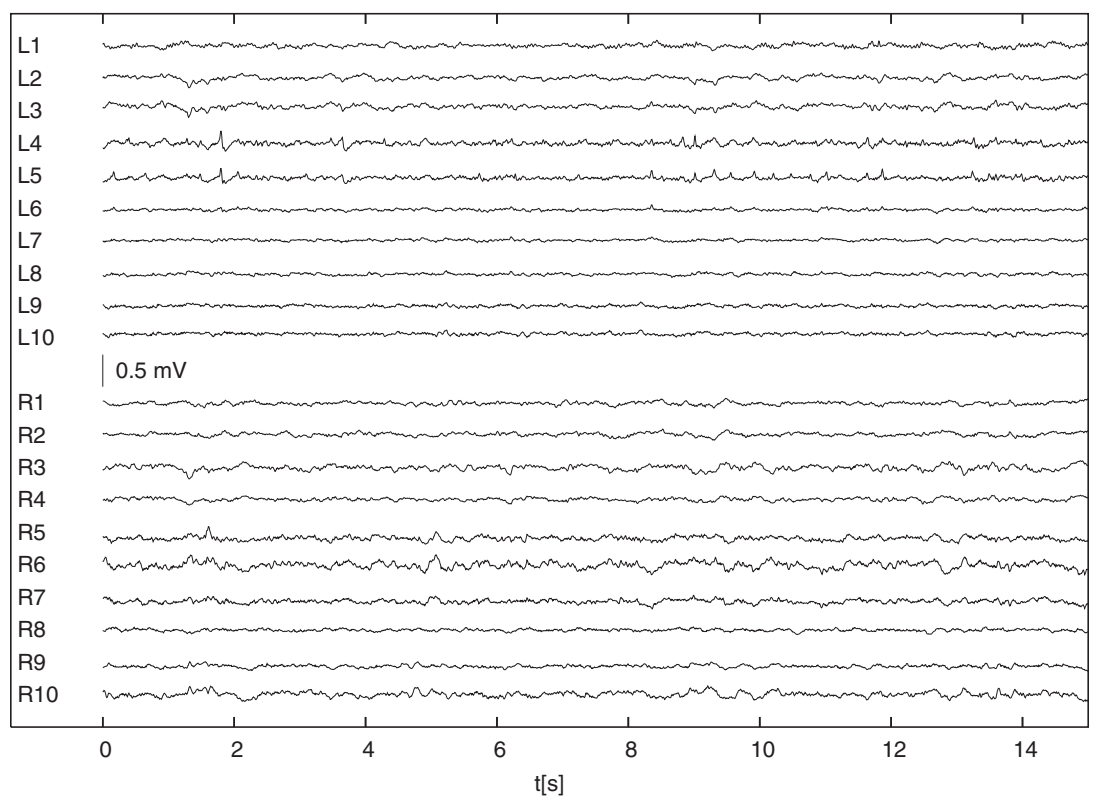

FIG. 6. Exemplary EEG recording from the seizure-free interval from patient 1. Upper part, recording from the left medial temporal lobe; lower part, recordings from the right medial temporal lobe. See Fig. 7 for electrode contact labeling.

\section{CONCLUSION}

The combined investigation of the directionality and interaction strength in networks of dynamical systems has revealed that separate analysis of these quantities may lead to spurious inference about underlying driving structures. For a coupled network of Rössler oscillators, we have demonstrated that an increasing level of local coupling strength renders a reliable detection of drivers increasingly difficult. Under such conditions, not only the detectability of coupling direction is impaired for high coupling strengths. In addition, for intermediate coupling strengths an apparent broadening of the driving region may be observed that may spuriously be interpreted as a driving subsystem. It should be emphasized that this spurious detection of driving may not be resolved using, e.g., confidence levels based on bootstrapping with surrogate techniques [41,68-70]. This is because the observed "bias", is not only caused by the intrinsic statistical properties (e.g., the mean and variance) of the measures, which in turn depend on intrinsic properties of the systems as
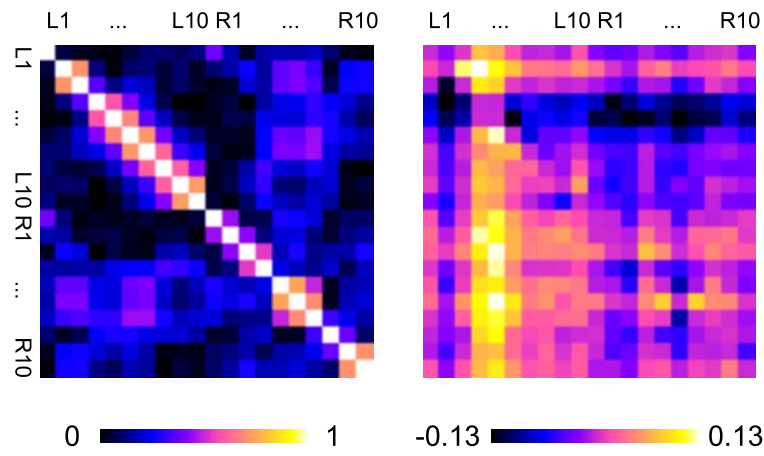

FIG. 7. (Color online) Mean phase coherence matrix $\mathbf{R}_{1}$ (right) and directionality matrix $\mathbf{D}_{1}$ for patient 1 . well as on the length of the time series. Instead, the crucial bias we investigate here is introduced by the involved couplings, and in practice, one may thus have to deal with a superposition of an intrinsic and an induced bias. Taking into account the strength of an interaction and the spectral characteristics of the signals may help to interpret the confidence levels obtained with surrogate techniques.

When investigating directional relationships in EEG time series from two epilepsy patients we observed driving regions in the focal hemisphere. The apparently broad driving region in one patient could possibly be caused by the high synchronization within the focal hemisphere. In this case, a misinterpretation through an exclusive analysis of the coupling direction could be avoided by including the strength of the coupling in the analysis. An important question is whether our findings can be attributed to the specific analysis techniques that were applied here, or whether they point to a principle limitation of the measurability of directionality. It
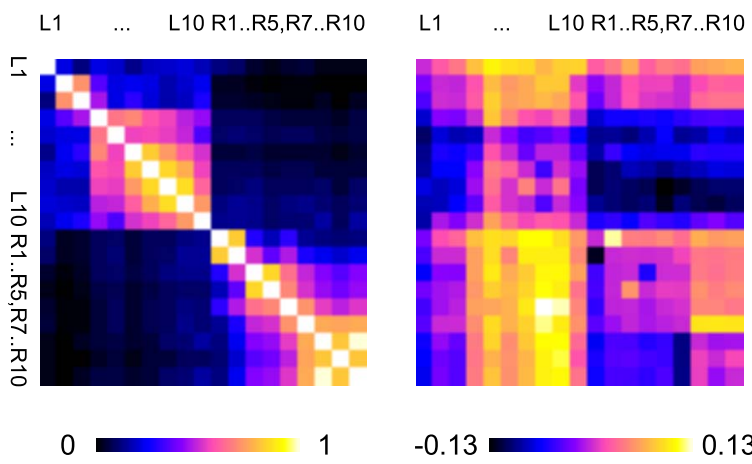

FIG. 8. (Color online) Mean phase coherence matrix $\mathbf{R}_{2}$ (right) and directionality matrix $\mathbf{D}_{2}$ for patient 2 . Note that only nine recording sites are present in the right hemisphere since the sixth contact of that electrode was not functional. 
has previously been discussed in Ref. [71] for the case of three coupled oscillators that applying bivariate techniques to pairs of time series taken from a multichannel recording does not necessarily allow one to identify the relevant information in the full data set. This may be achieved by multivariate time series analysis techniques [44-49] but it remains to be shown whether these techniques allow one to resolve interactions in systems where a driver is strongly coupled to one of two responders and weakly coupled to the other.

\section{ACKNOWLEDGMENTS}

The authors are grateful to Stephan Bialonski, Dieter Krug, and Matthäus Staniek for useful discussions and most valuable comments. This work was supported by the Deutsche Forschungsgemeinschaft (Contract No. SFB TR3 Subproject A2), by BONFOR, the intramural research fund of the University of Bonn and by the 6th Framework Programme of the European Commission (Marie Curie OIF).
[1] C. Huygens, Horologium Oscillatorium (Apud F. Muguet, Parisiis, France, 1673).

[2] R. L. Stratonovich, Topics in the Theory of Random Noise (Gordon and Breach, New York, 1963).

[3] A. T. Winfree, The Geometry of Biological Time (Springer, New York, 1980).

[4] H. Fujisaka and T. Yamada, Prog. Theor. Phys. 69, 32 (1983).

[5] Y. Kuramoto, Chemical Oscillations, Waves, and Tturbulence (Springer, New York, 1984).

[6] A. Pikovsky, Z. Phys. B: Condens. Matter 55, 149 (1984).

[7] V. S. Afraimovich, N. N. Verichev, and M. I. Rabinovich, Radiophys. Quantum Electron. 29, 795 (1986).

[8] I. I. Blekhman, Synchronization in Science and Technology (ASME Press, New York, 1988).

[9] L. M. Pecora and T. L. Carroll, Phys. Rev. Lett. 64, 821 (1990).

[10] A. Pikovsky, M. Rosenblum, and J. Kurths, Synchronization: A Universal Concept in Nonlinear Ssciences (Cambridge University Press, Cambridge, 2001).

[11] A. S. Pikovsky, Sov. J. Commun. Technol. Electron. 30, 85 (1985).

[12] J. F. Heagy, T. L. Carroll, and L. M. Pecora, Phys. Rev. E 50, 1874 (1994).

[13] A. K. Sood, J. Menendez, M. Cardona, and K. Ploog, Phys. Rev. Lett. 54, 2115 (1985).

[14] L. Fabiny, P. Colet, R. Roy, and D. Lenstra, Phys. Rev. A 47, 4287 (1993).

[15] R. Roy and K. S. Thornburg, Phys. Rev. Lett. 72, 2009 (1994).

[16] D. Y. Tang, R. Dykstra, M. W. Hamilton, and N. R. Heckenberg, Phys. Rev. E 57, 3649 (1998).

[17] D. W. Peterman, M. Ye, and P. E. Wigen, Phys. Rev. Lett. 74, 1740 (1995).

[18] E. Rosa, Jr., W. B. Pardo, C. M. Ticos, J. A. Walkenstein, and M. Monti, Int. J. Bifurcat. Chaos 10, 2551 (2000).

[19] T. L. Carroll and L. M. Pecora, Physica D 67, 126 (1993).

[20] L. Kocarev and U. Parlitz, Phys. Rev. Lett. 74, 5028 (1995).

[21] K. Pyragas, Phys. Lett. A 170, 421 (1992).

[22] N. F. Rulkov, L. S. Tsimring, and H. D. I. Abarbanel, Phys. Rev. E 50, 314 (1994).

[23] C. Schäfer, M. G. Rosenblum, J. Kurths, and H.-H. Abel, Nature (London) 392, 239 (1998).

[24] J. Buck and E. Buck, Science 159, 1319 (1968).

[25] C. J. Stam, Clin. Neurophysiol. 116, 2266 (2005).

[26] G. Buzsaki, Rhythms of the Brain (Oxford University Press, Oxford, 2006).

[27] M. G. Rosenblum, A. S. Pikovsky, and J. Kurths, Phys. Rev.
Lett. 78, 4193 (1997).

[28] M. G. Rosenblum, A. S. Pikovsky, and J. Kurths, Phys. Rev. Lett. 76, 1804 (1996).

[29] H. D. I. Abarbanel, N. F. Rulkov, and M. M. Sushchik, Phys. Rev. E 53, 4528 (1996).

[30] N. F. Rulkov, M. M. Sushchik, L. S. Tsimring, and H. D. I. Abarbanel, Phys. Rev. E 51, 980 (1995).

[31] S. J. Schiff, P. So, T. Chang, R. E. Burke, and T. Sauer, Phys. Rev. E 54, 6708 (1996).

[32] J. Arnhold, P. Grassberger, K. Lehnertz, and C. E. Elger, Physica D. 134, 419 (1999).

[33] F. Mormann, K. Lehnertz, P. David, and C. E. Elger, Physica D. 144, 358 (2000).

[34] C. W. J. Granger, Econometrica 37, 424 (1969).

[35] J. P. Pijn, P. C. Vijn, F. H. Lopes da Silva, W. Van Ende Boas, and W. Blanes, Neurophysiol. Clin. 20, 1 (1990).

[36] T. Schreiber, Phys. Rev. Lett. 85, 461 (2000).

[37] M. G. Rosenblum and A. S. Pikovsky, Phys. Rev. E 64, 045202(R) (2001).

[38] D. A. Smirnov and B. P. Bezruchko, Phys. Rev. E 68, 046209 (2003)

[39] D. A. Smirnov and R. G. Andrzejak, Phys. Rev. E 71, 036207 (2005).

[40] D. Smirnov, B. Schelter, M. Winterhalder, and J. Timmer, Chaos 17, 013111 (2007).

[41] M. Palus and M. Vejmelka, Phys. Rev. E 75, 056211 (2007).

[42] J. Waddell, R. Dzakpasu, V. Booth, B. Riley, J. Reasor, G. Poe, and M. Zochowski, J. Neurosci. Methods 162, 320 (2007).

[43] T. Kiemel, K. Gormley, L. Guan, T. Williams, and A. Cohen, J. Comput. Neurosci. 15, 233 (2003).

[44] C. Allefeld and J. Kurths, Int. J. Bifurcat Chaos 14, 417 (2004).

[45] K. J. Blinowska, R. Kuś, and M. Kamiński, Phys. Rev. E 70, 050902(R) (2004).

[46] M. Müller, G. Baier, A. Galka, U. Stephani, and H. Muhle, Phys. Rev. E 71, 046116 (2005).

[47] S. Bialonski and K. Lehnertz, Phys. Rev. E 74, 051909 (2006).

[48] M. Müller, K. Wegner, U. Kummer, and G. Baier, Phys. Rev. E 73, 046106 (2006).

[49] B. Schelter, M. Winterhalder, R. Dahlhaus, J. Kurths, and J. Timmer, Phys. Rev. Lett. 96, 208103 (2006).

[50] M. G. Rosenblum, L. Cimponeriu, A. Bezerianos, A. Patzak, and R. Mrowka, Phys. Rev. E 65, 041909 (2002).

[51] B. Bezruchko, W. Ponomarenko, M. G. Rosenblum, and A. S. Pikovksy, Chaos 13, 179 (2003).

[52] D. Gabor, Proc. IEEE 93, 429 (1946). 
[53] P. Panter, Modulation, Noise, and Spectral Analysis (McGrawHill, New York, 1965).

[54] T. Kiemel and A. Cohen, J. Comput. Neurosci. 5, 267 (1998).

[55] O. E. Rössler, Phys. Lett. 57A, 397 (1976).

[56] H. Osterhage, F. Mormann, T. Wagner, and K. Lehnertz, Int. J. Neural Syst. 17, 139 (2007).

[57] H. Daido, Phys. Rev. Lett. 77, 1406 (1996).

[58] F. Mormann, R. G. Andrzejak, T. Kreuz, C. Rieke, P. David, C. E. Elger, and K. Lehnertz, Phys. Rev. E 67, 021912 (2003).

[59] F. Mormann, T. Kreuz, C. Rieke, R. G. Andrzejak, A. Kraskov, P. David, C. E. Elger, and K. Lehnertz, Clin. Neurophysiol. 116, 569 (2005).

[60] M. Le Van Quyen, J. Soss, V. Navarro, R. Robertson, M. Chavez, M. Baulac, and J. Martinerie, Clin. Neurophysiol. 116, 559 (2005).

[61] S. Kalitzin, D. Velis, P. Suffczynski, J. Parra, and F. Lopes da Silva, Clin. Neurophysiol. 116, 718 (2005).

[62] J. P. Pijn and F. H. Lopes da Silva, in Basic Mechanisms of the EEG, Brain Dynamics, edited by S. Zschocke and E. J. Speckmann (Birkhauser, Boston, 1993), pp. 41-61.
[63] P. J. Franaszczuk and G. K. Bergey, Brain Topogr. 11, 13 (1998).

[64] F. Wendling, F. Bartolomei, J. J. Bellanger, and P. Chauvel, Clin. Neurophysiol. 112, 1201 (2001).

[65] F. Bartolomei, F. Wendling, J. J. Bellanger, J. Regis, and P. Chauvel, Clin. Neurophysiol. 112, 1746 (2001).

[66] D. A. Smirnov, M. B. Bodrov, J. L. Perez Velazquez, R. A. Wennberg, and B. P. Bezruchko, Chaos 15, 024102 (2005).

[67] M. Paluš, V. Komàrek, Z. Hrnčǐr, and K. Štěrbová, Phys. Rev. E 63, 046211 (2001).

[68] R. G. Andrzejak, A. Kraskov, H. Stögbauer, F. Mormann, and T. Kreuz, Phys. Rev. E 68, 066202 (2003).

[69] M. Paluš and A. Stefanovska, Phys. Rev. E 67, 055201(R) (2003).

[70] R. Andrzejak, A. Ledberg, and G. Deco, New J. Phys. 8, 6 (2006).

[71] L. Cimponeriu, M. G. Rosenblum, T. Fieseler, J. Dammers, M. Schiek, M. Majtanik, P. Morosan, A. Bezerianos, and P. A. Tass, Prog. Theor. Phys. Suppl. 150, 22 (2003). 